\title{
Development of a Freeze-Dried Skin Care Product Composed of Hyaluronic Acid and Poly( $\gamma$-Glutamic Acid) Containing Bioactive Components for Application after Chemical Peels
}

\author{
Yuka Isago, Ryusuke Suzuki, Eri Isono, Yuya Noguchi, Yoshimitsu Kuroyanagi* \\ R\&D Center for Artificial Skin, School of Allied Health Sciences, Kitasato University, Sagamihara, Japan \\ Email: "kuroyana@ahs.kitasato-u.ac.jp
}

Received 13 July 2014; revised 10 August 2014; accepted 20 August 2014

Copyright (C) 2014 by authors and Scientific Research Publishing Inc.

This work is licensed under the Creative Commons Attribution International License (CC BY). http://creativecommons.org/licenses/by/4.0/

(c) (i) Open Access

\section{Abstract}

Eight types of spongy sheet were prepared by freeze-drying aqueous solutions of hyaluronic acid (HA) and poly $(\gamma$-glutamic acid) (PGA) with or without bioactive components including vitamin C derivative (VC), glucosylceramide (GC), and epidermal growth factor (EGF). Spongy sheets were categorized into the following groups: Group I (HA/PGA), Group II (HA/PGA + VC), Group III (HA/PGA + GC), Group IV (HA/PGA + VC, GC), Group V (HA/PGA + EGF), Group VI (HA/PGA + VC, EGF), Group VII (HA/PGA + GC, EGF), and Group VIII (HA/PGA + VC, GC, EGF). In the first experiment, we examined fibroblast proliferation in conditioned medium that had been prepared by immersing each spongy sheet in a conventional culture medium. EGF-incorporating spongy sheets (Groups V-VIII) enhanced fibroblast proliferation more than EGF-free spongy sheets (Groups I-IV). In the second experiment, cytokine production by fibroblasts was evaluated using a wound surface model. This involved elevation of fibroblasts-incorporating collagen gel sheets to the air-liquid interface, on which a spongy sheet (Groups I, IV, V and VIII) was placed and cultured for 1 week. EGF-incorporating spongy sheets (Groups V and VIII) enhanced the production of vascular endothelial growth factor (VEGF) and hepatocyte growth factor (HGF) by fibroblasts more than EGF-free spongy sheets (Groups I and IV). The effect of these four types of spongy sheet on wounds was investigated in animal experiments. Chemical peel was performed by contacting $50 \%$ trichloroacetic acid (TCA) on the dorsal region of mice, after which a spongy sheet was placed, and the wound condition was then observed in a two-week period. Angiogenesis was facilitated to a

\footnotetext{
*Corresponding author.
} 
greater degree in Group VIII compared with Groups I, IV and V. This finding indicates that Group VIII spongy sheet is a promising aid for skin recovery after chemical peel.

\author{
Keywords \\ Chemical Peel, Skin Care Product, Hyaluronic Acid, Poly( $\gamma$-Glutamic Acid), Vitamin C Derivative, \\ Glucosylceramide, Epidermal Growth Factor
}

\title{
1. Introduction
}

Our skin performs many functions. The skin is composed of three layers: the epidermis, the dermis and the subcutis. There is a distinct interface between the epidermis and dermis, whereas the dermis merges with the subcutis without a clear boundary. Moving from the surface to the interior, there are five layers of the epidermis: the horny layer, the clear layer, the granular layer, the prickle cell layer, and the basal layer. The two lowest cell layers consist of living cells that replenish the cells that are shed from the horny layer. The dermis is composed of two different regions: the papillary dermis and the reticular dermis. Fine vascular loops that provide nutrient supply to the epidermis are interspersed in the papillary dermis. The main cell in connective tissue is the fibroblast. Collagen fibers are interspersed in the reticular layer. Many different types of injury result in damage to the skin. However, the skin is capable of restoring the original protective covering by using repair mechanisms in the individual layers. Based on the regenerative potential of the skin, chemical peel is a cosmetic procedure using a chemical agent that has been widely used in the field of aesthetic dermatology and aesthetic reconstructive surgery [1]-[8]. This procedure involves the application of a chemical agent that results in exfoliation of the skin followed by regrowth of new skin. This leads to skin rejuvenation. The treatment is classified into superficial, medium and deep peels. A superficial peel causes damage to the epidermis and the epidermal-dermal interface. A medium peel causes damage to the papillary dermis. A deep peel causes damage to the reticular dermis. Typical chemical agents are $10 \%-30 \%$ trichloroacetic acid (TCA) or 70\% glycolic acid for superficial peels, 50\% TCA for medium peels, and phenol or Baker-Gordon formula for deep peels [4]. Chemical peel can improve the skin condition when used properly but there is a risk of complications. In general, deeper peels require longer healing times and are more likely to result in a larger number of complications. Therefore, the benefit-to-risk ratio must be considered in the selection of the chemical peel. Although chemical peels resulted in chemical burns, most physicians did not focus on the importance of skin care products after chemical peels. In addition to choosing the appropriate peeling agent and their application period, the physician must choose the appropriate skin care product for application after chemical peels to obtain a satisfactory clinical result. The most critical issue is how to facilitate wound healing after chemical peels. Successful management of a corrosive wound caused by chemical peels requires the use of excellent skin care products that are capable of facilitating wound healing. This includes epithelialization, angiogenesis, and creation of a moist environment for re-epithelialization. Therefore, the skin care product should have wound-healing and moisturizing effects.

We have developed several types of wound dressing composed of hyaluronic acid (HA) and collagen (Col), which contain bioactive substances such as magnesium ascorbyl phosphate (vitamin C derivative: VC) and epidermal growth factor (EGF) [9] [10]. HA is considered an important biomaterial for wound healing because it is a major extracellular matrix component. Because of its unique hygroscopic, rheological and viscoelastic properties, HA creates an excellent wound-healing environment. High molecular weight HA (HMW-HA) provides an excellent wound-healing environment, while low molecular weight HA (LMW-HA) induces angiogenesis [11]-[14]. It is known that EGF is beneficial for wound healing because of its effects on proliferation of keratinocytes, fibroblasts and vascular endothelial cells, thus facilitating the formation of granulation tissue and reepithelialization [15] [16]. In addition, EGF can stimulate fibroblasts to synthesize an increased amount of vascular endothelial growth factor (VEGF) and hepatocyte growth factor (HGF) [17]. VEGF and HGF are potent cytokines that promote angiogenesis. Recent research has demonstrated that simultaneous administration of VEGF and HGF synergistically promotes new blood vessel formation compared with administration of each factor alone [18]. In addition, HGF is considered to be one of the key cytokines involved in epithelialization in addition to angiogenesis [19]. Thus, EGF is a promising factor for wound healing. Vitamin C stimulates colla- 
gen synthesis by fibroblasts, and also has antioxidant effects. The combination use of EGF and VC has a synergistic effect of enhancing HGF production by fibroblasts in vitro [20]. Therefore, VC is also a promising factor for wound healing. In addition, poly( $\gamma$-glutamic acid) (PGA) and glucosylceramide (GC) have moisturizing effects in the epidermis. Based on our development of various types of wound dressing, the present study aims to develop a freeze-dried spongy sheet composed of HA and PGA, which contains various bioactive components including VC, GC, and EGF. The potential benefit of these products was evaluated in cultures and in animal experiments using nude mice.

\section{Materials and Methods}

\subsection{Experimental Materials}

HA (Bio Sodium Hyaluronate HA 20) was purchased from Shiseido (Tokyo, Japan). PGA and GC were purchased from Ichimaru Pharcos (Gifu, Japan). VC was purchased from Showa Denko (Tokyo, Japan). Recombinant human EGF was purchased from Shanghai Haohai Biological Technology (Shanghai, China). Collagen (NMP Collagen PS) was purchased from Nippon Meat Packers (Osaka, Japan). Polyurethane film dressing (Bioclusive) and Elastic tape (Elastikon) were purchased from Johnson \& Johnson (New Jersey, USA).

\subsection{Preparation of the Skin Care Product}

The spongy sheet was prepared using a similar method described in our previous article [20]. Briefly, HA power (4 g) was dissolved in $400 \mathrm{~mL}$ of distilled water (DW) to prepare a HMW-HA (molecular weight, $2000 \mathrm{kDa}$ ) solution. HA powder $(16 \mathrm{~g})$ was dissolved in $1600 \mathrm{~mL}$ of DW, which was then autoclaved at $120^{\circ} \mathrm{C}$ for $1 \mathrm{~h}$ to prepare partially hydrolyzed LMW-HA (molecular weight, $150 \mathrm{kDa}$ ) solution. PGA powder (4 g) was dissolved in $400 \mathrm{~mL}$ of DW to prepare a PGA solution. These three solutions were mixed to give a HA/PGA solution $(2400 \mathrm{~mL})$ and then the $\mathrm{pH}$ was adjusted to 7 . This mixed solution was divided into two portions $(1200 \mathrm{~mL} \times 2)$. EGF (1 mg) was dissolved in $10 \mathrm{~mL}$ of DW, and it was then added to a half-mixed solution to create EGF-incorporating HA/PGA solution, while $10 \mathrm{~mL}$ of DW was added to another half-mixed solution to create an EGFfree HA/PGA solution. EGF-free HA/PGA solution was divided into four portions (300 mL $\times 4$ ). EGF-incorporating HA/PGA solution was also divided into four portions $(300 \mathrm{~mL} \times 4)$. DW $(30 \mathrm{~mL})$ alone, $\mathrm{DW}(30 \mathrm{~mL})$ containing VC $(0.5 \mathrm{~g})$, DW $(30 \mathrm{~mL})$ containing GC $(0.5 \mathrm{~g})$, or DW $(30 \mathrm{~mL})$ containing VC $(0.5 \mathrm{~g})$ and GC (0.5 g) was added to the EGF-free HA/PGA solution to create four solutions: Group I (HA/PGA solution only), Group II (+VC), Group III (+GC), Group IV (+VC, GC). Four other solutions were prepared in a similar manner: Group V (+EGF), Group VI (+VC, EGF), Group VII (+GC, EGF), and Group VIII (+VC, GC, EGF). Each mixed solution $(33 \mathrm{~mL})$ was poured into a tray $(5 \mathrm{~cm} \times 8 \mathrm{~cm})$, and then refrigerated at $44^{\circ} \mathrm{C}$ for $2 \mathrm{~h}$, followed by freezing at $-85^{\circ} \mathrm{C}$ and freeze-drying to obtain eight types of spongy sheet: EGF-free spongy sheet (Groups I-IV) and EGF-incorporating spongy sheet (Groups V-VIII). Each product was packed in a bag and kept in a dry sterilizer at $110^{\circ} \mathrm{C}$ for $1 \mathrm{~h}$.

\subsection{Experiments in Vitro}

\subsubsection{Fibroblast Proliferation in Conditioned Media}

Fibroblasts were harvested from a piece of human dermis, expanded and cryopreserved to obtain a working cell stock as described previously [17]. When required, cryopreserved cells were thawed and cultured for two passages in culture medium (Dulbecco's modified Eagle's medium [DMEM] supplemented with 10\% fetal bovine serum [FBS]) to obtain an adequate number of cells. Conditioned medium was prepared by immersing a piece of spongy sheet $(5 \mathrm{~cm} \times 4 \mathrm{~cm})$ in $50 \mathrm{~mL}$ of culture medium for 1 day at $37^{\circ} \mathrm{C}$. Fibroblasts were seeded into 20 flasks $\left(75 \mathrm{~cm}^{2}\right)$ at a density of $1 \times 10^{4}$ cells/ $\mathrm{cm}^{2}$, and cultured in the culture medium $(15 \mathrm{~mL})$. The density of attached cells after 1 day was calculated using a conventional method using 2 flasks. Cell proliferation was assessed using 18 flasks under 9 culture conditions: in culture medium (referred to as control medium) and in each conditioned medium (Groups I-VIII). After 1 day of cultivation, the culture medium was replaced with either control medium $(20 \mathrm{~mL})$ or one of the conditioned media $(20 \mathrm{~mL})$. Cell density at days 4 and 7 was measured using 2 flasks for each group. The concentrations of VC, GC and EGF in each spongy sheet (Groups IV-VIII) were $50 \mathrm{mg} / 40 \mathrm{~cm}^{2}, 50 \mathrm{mg} / 40 \mathrm{~cm}^{2}$, and $25 \mu \mathrm{g} / 40 \mathrm{~cm}^{2}$, respectively (expressed as $\mathrm{mg} / \mathrm{cm}^{2}$ and $\mu \mathrm{g} / \mathrm{cm}^{2}$ for conven- 
ience instead of $\mathrm{mg} / \mathrm{cm}^{3}$ and $\mu \mathrm{g} / \mathrm{cm}^{3}$ ). Each conditioned medium (Groups I-VIII) contained VC and/or GC at concentrations of $10 \mathrm{mg} / 20 \mathrm{~mL}$. In addition, four types of conditioned medium (Groups IV-VIII) contained EGF at a concentration of $5 \mu \mathrm{g} / 20 \mathrm{~mL}$. The cell proliferation experiment was conducted four times $(n=4)$.

\subsubsection{Fibroblast Metabolic Activity and Cytokine Production in a Wound Surface Model}

Cultured dermal substitute (CDS) was prepared as described previously [17]. Col powder was sterilized using a vacuum drying oven at $121^{\circ} \mathrm{C}$ for $2 \mathrm{~h}$. Col powder was dissolved in sterilized distilled water at a concentration of $1 \%$. A concentrated DMEM was prepared at a double concentration of conventional one. Fibroblasts were suspended in double-concentrated DMEM supplemented with $20 \%$ FBS at a cell density of $80 \times 10^{5}$ cells/12mL. Fibroblast suspensions $(12 \mathrm{~mL})$ were mixed with $12 \mathrm{~mL}$ of $1 \%$ sterile Col solution at a temperature below $10^{\circ} \mathrm{C}$. The mixture was then poured into a dish $(8 \mathrm{~cm} \times 5 \mathrm{~cm})$. A sheet of nylon mesh $(8 \mathrm{~cm} \times 5 \mathrm{~cm})$ was incorporated into the mixture to provide mechanical support. The mixture was placed in a $5 \% \mathrm{CO}_{2}$ incubator at $37^{\circ} \mathrm{C}$ for 1 day to promote jellification. The seeding density of fibroblasts was $2 \times 10^{5} \mathrm{cells} / \mathrm{cm}^{2}$ (expressed as cells $/ \mathrm{cm}^{2}$ for convenience instead of cells $\left./ \mathrm{cm}^{3}\right)$. The fibroblast-incorporating Col gel was moved to a polystyrene dish $(10 \mathrm{~cm}$ $\times 10 \mathrm{~cm}$ ) and cultured in $50 \mathrm{~mL}$ culture medium for 6 days to obtain the CDS. The CDS was cut into sizes of 4 $\mathrm{cm} \times 5 \mathrm{~cm}$ and then placed on a stainless mesh in a polystyrene dish $(10 \mathrm{~cm} \times 10 \mathrm{~cm})$, followed by the addition of culture medium $(60 \mathrm{~mL})$. The CDS was elevated to the air-culture medium interface to prepare a wound surface model, on which one type of spongy sheet was placed, and cultured for 7 days. In the control group, no wound dressing was placed on the CDS. After cultivation for 7 days, the cell metabolic activity and VEGF and HGF production in the CDS were measured using the MTT assay and ELISA, respectively, according to the methods described in our previous article [17]. These experiments were conducted four times $(n=4)$.

\subsection{Experiments in Vivo}

\subsubsection{Animal Experiment: Chemical Peel with 50\% TCA on the Dorsum of Nude Mice}

The dorsal region of nude mice (BALB/cAJcl-nu, male, 8 weeks of age, CLEA Japan Inc., Tokyo, Japan) was shaved under anesthesia and the left dorsal region was treated with gauze $(10 \mathrm{~mm} \times 15 \mathrm{~mm}$, 8 sheets $)$ containing $50 \%$ TCA $(150 \mu \mathrm{L})$. Specifically, this procedure involved contact with 50\% TCA-soaked gauze for $1 \mathrm{~min}$, followed by removal of the gauze, air exposure for $19 \mathrm{~min}$, and a rinse with sterilized DW (1.5 mL). A spongy sheet $(10 \mathrm{~mm} \times 15 \mathrm{~mm})$ was placed onto this chemical burn area, and sterilized DW $(200 \mu \mathrm{L})$ was added to hydrate the spongy sheet. A polyurethane film dressing (Bioclusive) was placed on the hydrated spongy sheet, on which gauze was placed and fixed with elastic tape (Elastikon). In the control group, the same procedure was performed without placement of a spongy sheet. This procedure was repeated at postoperative days 3, 7, and 10 . The wound-healing process was evaluated by examining the macroscopic appearance and histology at postoperative day 14 . These experiments were conducted using four mice $(n=4)$. The animal study complied with the guidelines of the Animal Study Committee of the School of Allied Health Sciences, Kitasato University.

\subsubsection{Histological Evaluation of Angiogenesis}

Biopsy specimens were fixed in 10\% formalin neutral buffer solution, embedded in paraffin, sectioned at $4 \mu \mathrm{m}$ thickness and mounted onto slides. Serial sections were deparaffinized and stained with hematoxylin and eosin (HE). Histological observation was conducted at three positions: 1) middle of the cranial wound margin and wound center, 2) wound center, and 3) middle of the caudal wound margin and wound center. Specimens were then observed by light microscopy. The extent of wound edema was measured by tracing out the cross-sectional area of fluid accumulation in a region measuring $500 \mu \mathrm{m} \times 500 \mu \mathrm{m}$ on photographs of HE-stained samples, which was analyzed using an imaging software (Scion Image, Scion Corporation). In a similar manner, the extent of angiogenesis was measured by tracing out the cross-sectional area of blood vessels in a region measuring $500 \mu \mathrm{m} \times 500 \mu \mathrm{m}$ on photographs of HE-stained samples, followed by analysis using imaging software. These observations were conducted at twelve positions $(n=4 \times 3)$.

\subsection{Statistical Evaluation}

Data are expressed as means \pm standard error (SE). Statistical analysis was performed using the Tukey-Kramer test. 


\section{Result}

\subsection{Preparation of the Skin Care Product}

Macroscopic view and scanning electron microphotograph of the EGF-incorporating wound dressing are shown in Figure 1. The spongy sheet showed a unique cross-sectional profile displaying vertical holes.

\subsection{Fibroblast Proliferation in Conditioned Media}

Fibroblast proliferation in conditioned and control media is shown in Figure 2. The fibroblast density in conditioned media with EGF (Groups V-VIII) was almost two times greater than those in conditioned media without EGF (Groups I-VI) and control medium. There was no difference in fibroblast proliferation among the four conditioned media with EGF (Groups V-VIII). In addition, there was no difference among the four conditioned media without EGF (Groups I-IV). No difference was seen between the four conditioned media without EGF (Groups I-IV) and the control medium.

\subsection{Fibroblast Metabolic Activity and Cytokine Production in a Wound Surface Model}

The CDS was elevated to the air-culture medium interface to prepare a wound surface model (Figure 3), on which one type of spongy sheet was placed, and cultured for 7 days. Figure 4 shows the optical density (OD) values of the MTT assay that corresponds to the metabolic activity and proliferation of fibroblasts in the CDS after 7 days of cultivation in the wound surface model. The OD value was slightly lower in Group I compared with the control group (no spongy sheet). This is likely due to partial absorption of medium from the upper layer of CDS into the spongy sheet and thus, this might have limited the nutrient supply to fibroblasts in Group I. However, there was no significant difference in the OD value between Group I and the control group. The OD value in Group IV was significantly higher than that of Group I. This indicates that the mixture of VC and GC increased metabolic activity. The OD value of Group V was significantly higher than that of Group I. This indicates that EGF promotes metabolic activity and cell proliferation. The OD value was significantly higher in Group VIII compared with Group VI. This also suggests that EGF promotes metabolic activity and cell proliferation. The OD value was higher in Group VIII compared with Group V. This increase in OD value appeared to be due to the combined effects of VC, GC and EGF.
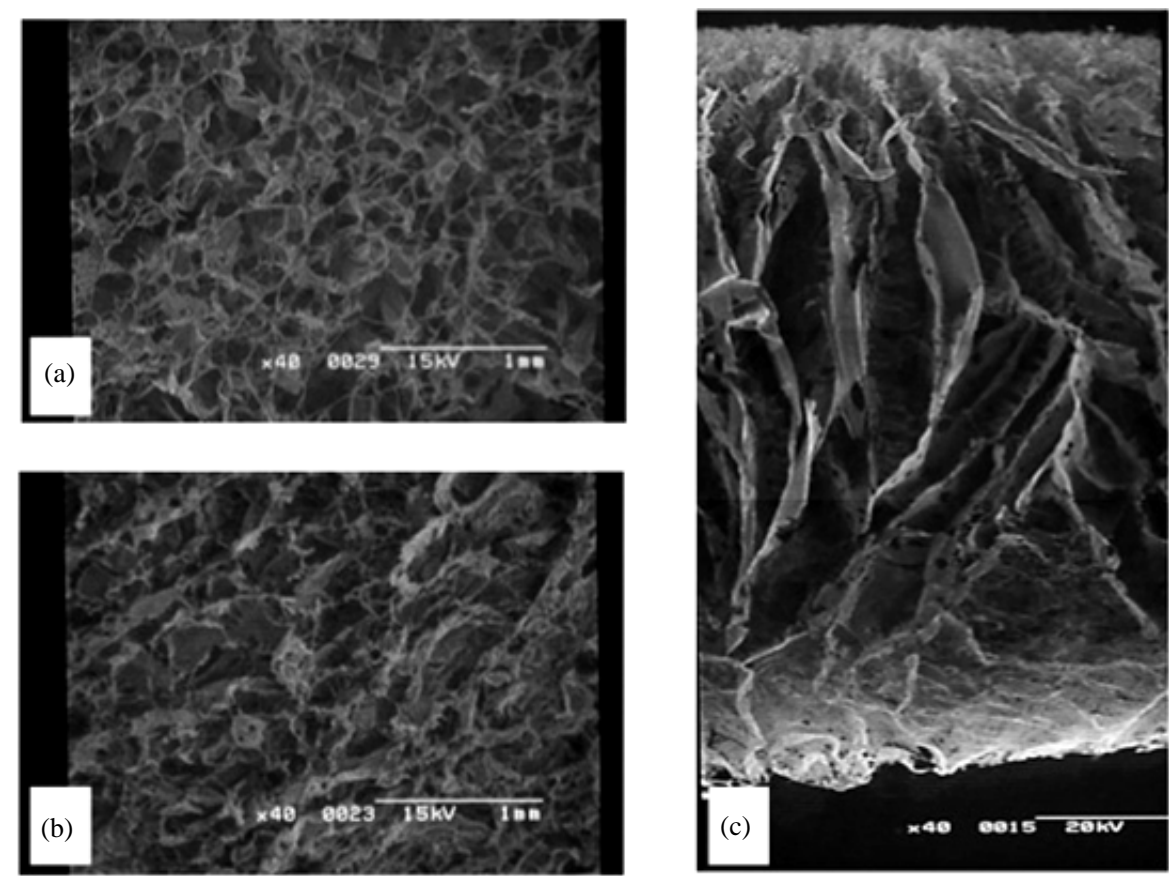

Figure 1. Scanning electron microphotographs of the skin care product (Group I): top surface (a); bottom surface (b); and cross-section (c). Scale bar is $1 \mathrm{~mm}$. 


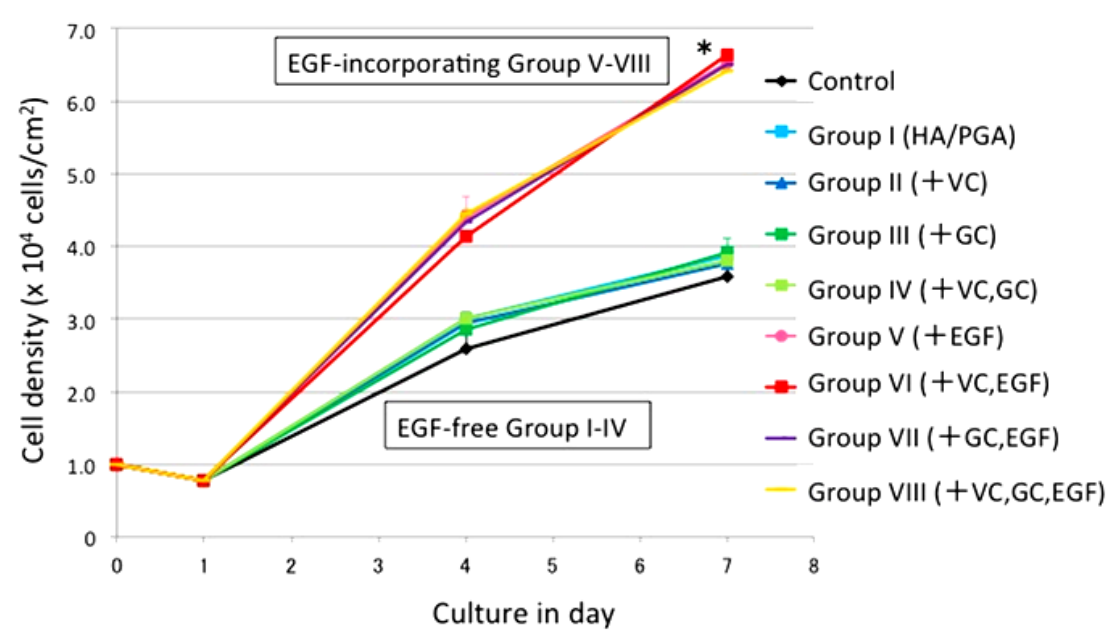

Figure 2. Fibroblast proliferation in culture medium and in each conditioned medium. $\mathrm{n}=4 .{ }^{*} \mathrm{P}<0.01$ vs. EGF-free Groups I-IV.

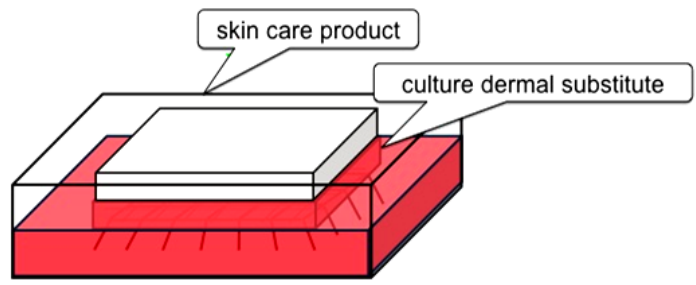

Figure 3. Wound surface model: CDS was elevated to the air-culture medium interface, on which skin care product was placed, and cultured for 7 days.

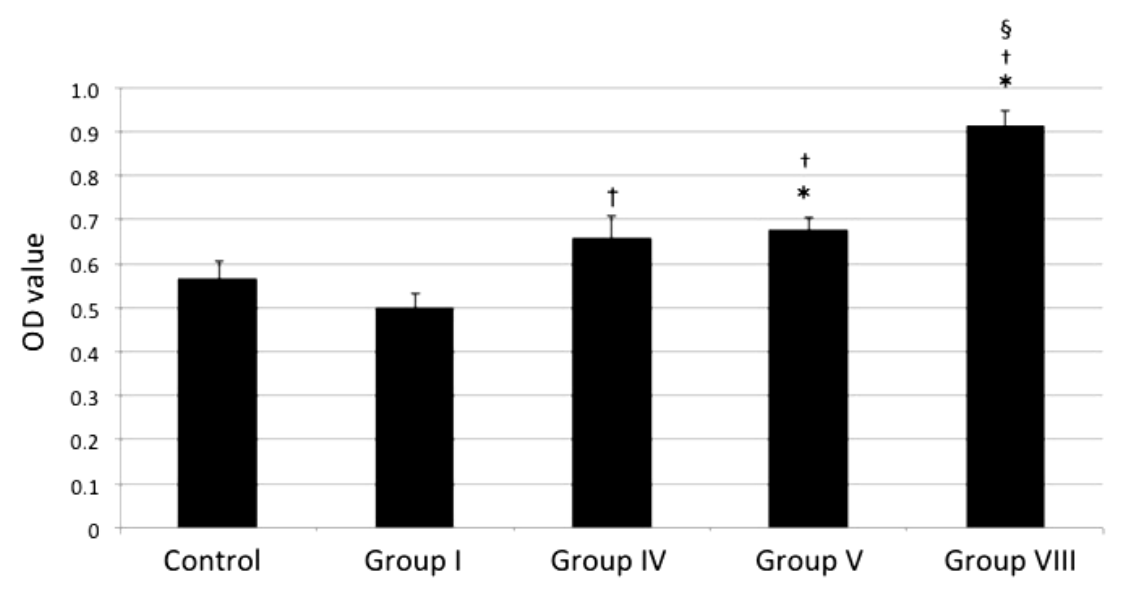

Figure 4. OD values that correspond to the metabolic activity of fibroblasts in the CDS after 7 days of cultivation at the air-medium interface: control group (no spongy sheet), Group I (HA/PGA), Group IV (HA/PGA + VC, GC), Group V (HA/PGA + EGF), and Group VIII (HA/PGA + VC, GC, EGF), as measured by the MTT assay. $n=4 .{ }^{*} P<$ 0.01 vs. Control. ${ }^{\dagger} P<0.01$ vs. Group I. ${ }^{\S} P<0.01$ vs. Group IV.

Figure 5 shows VEGF production by fibroblasts in the CDS after 7 days of cultivation in the wound surface model. VEGF production in Group IV was lower than that in Group I. This suggests that the mixture of VC and GC has no effect on VEGF production. VEGF production in Group V was significantly higher than that in Group I. This suggests that EGF released from the spongy sheet can enhance VEGF production. VEGF production was significantly higher in Group VIII compared with Group IV. This also suggests that EGF promotes 


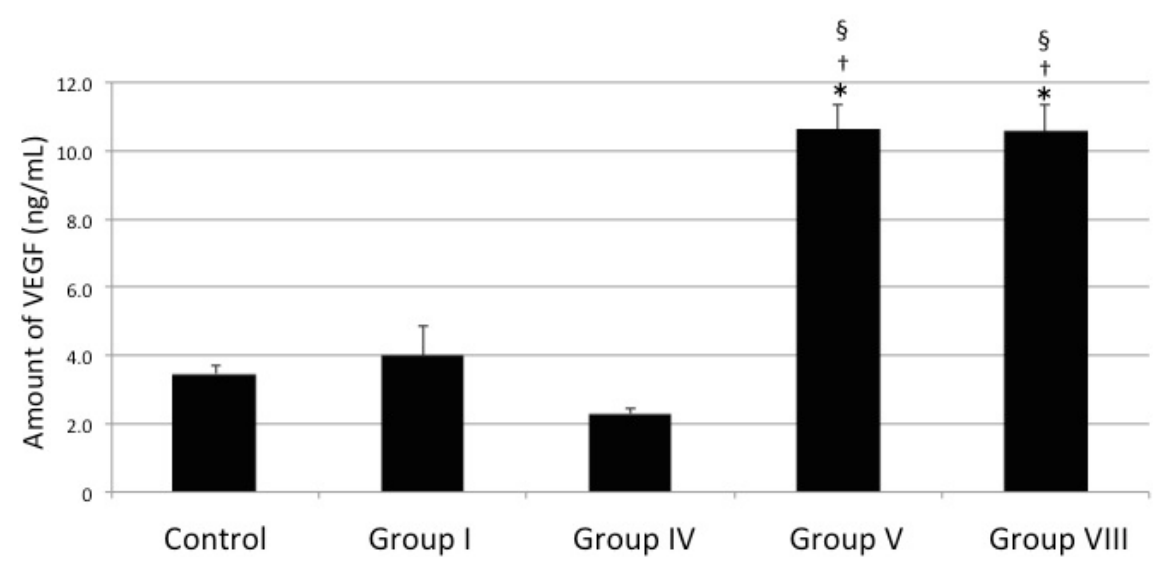

Figure 5. VEGF production from fibroblasts in the CDS after 7 days of cultivation at the air-medium interface: control group, Group I, Group IV, Group V and Group VIII, as measured by ELISA. $\mathrm{n}=4 .{ }^{*} P<0.01$ vs. Control. ${ }^{\dagger} P<0.01$ vs. Group I. ${ }^{\S} P<0.01$ vs. Group IV.

VEGF production. There was no difference between Group V and VIII. This also suggests that the mixture of VC and GC has no effect on VEGF production.

Figure 6 shows HGF production by fibroblasts in the CDS after 7 days of cultivation in the wound surface model. There was no significant difference in HGF production between Group I and Group IV. This suggests that the mixture of VC and GC has no effect on HGF production. HGF production in Group V was significantly higher than that in Group I. This suggests that EGF released from the spongy sheet can more effectively enhance HGF production. HGF production was significantly higher in Group VIII compared with Group IV. This also suggests that EGF promotes HGF production. HGF production was higher in Group VIII compared with Group V. This increase in HGF production appeared to be due to the combined effects of VC, GC and EGF.

\subsection{Chemical Peel with 50\% TCA on the Dorsum of Nude Mice}

Figure 7 shows the macroscopic appearances of the wound caused by application of 50\% TCA. After 50\% TCA-soaked gauze was placed on the skin for 1 min followed by removal of the gauze, the wound initially showed a slightly whitish color. After exposure to air for $19 \mathrm{~min}$, the wound showed a distinct white color. A spongy sheet placed on the wound area became a highly hydrated layer after absorbing DW, and a polyurethane film dressing (Bioclusive) was placed to protect the chemical peel area.

Figure 8 shows the macroscopic appearances of the wound at postoperative day 14. The necrotic tissue caused by contact with TCA had formed a dry scab in the control group (no spongy sheet) at postoperative day 14. In contrast, in four groups (Groups I, IV, V and VIII), the majority of the scab had disappeared at postoperative day 14, and the wound condition was improved.

Figure 9 shows the histological appearance of the wounds at postoperative day 14. The thickness of granulation tissue in four groups (Groups I, IV, V and VIII) was thicker than that in the control group. Granulation tissue formation with angiogenesis was facilitated in these four groups compared with the control group. Figure 10 shows the total area of edema. There was no significant difference among the groups (Groups I, IV, V and VIII). However, the total area of edema decreased in the four groups compared with the control group. Figure 11 shows the total area of angiogenesis. Angiogenesis was facilitated in the four groups (Groups I, IV, V and VIII) compared with the control group. In particular, angiogenesis was significantly facilitated in Group VIII compared with the control group. The animal experiment results indicate that a spongy sheet composed of HA and PGA potentially enhances wound healing, and the incorporated VC, GC and EGF potentially increases the wound-healing effect of the spongy sheet.

\section{Discussion}

When strong acids come into contact with the skin, there results a deep corrosive wound to the skin. The wound conditions vary depending on the concentration of the acid and the duration of exposure. Since this skin damage 


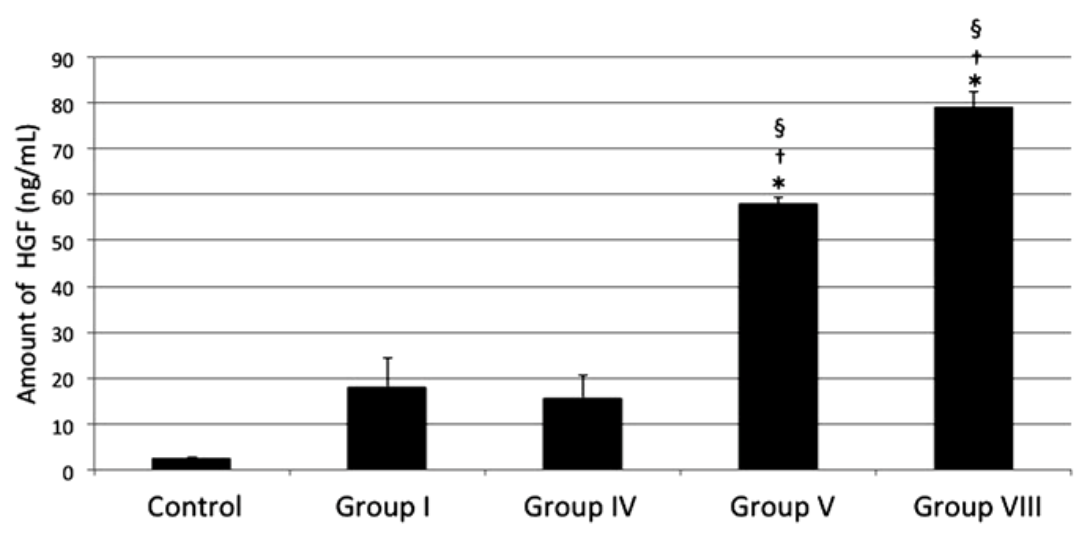

Figure 6. HGF production from fibroblasts in the CDS after 7 days of cultivation at the air-medium interface: control group, Group I, Group IV, Group V and Group VIII, as measured by ELISA. $n=4 .{ }^{*} P<0.01$ vs. Control. ${ }^{\dagger} P<0.01$ vs. Group I. $^{\S} P<$ 0.01 vs. Group IV.

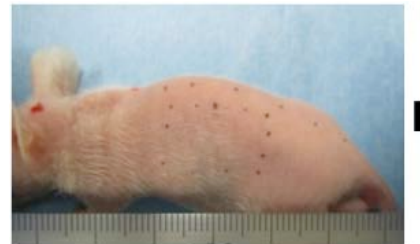

(a)

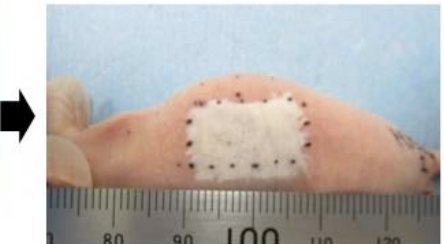

(b)

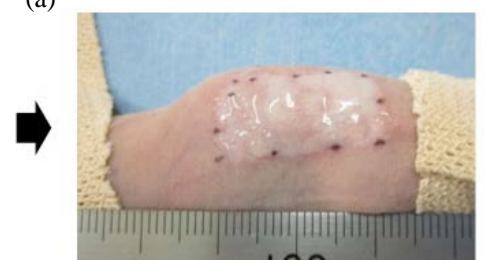

(c)

Figure 7. Macroscopic appearances of the skin surface after chemical peel using 50\% TCA: (a) before chemical peel, (b) after chemical peel using 50\% TCA, (c) hydrated spongy sheet with DW.

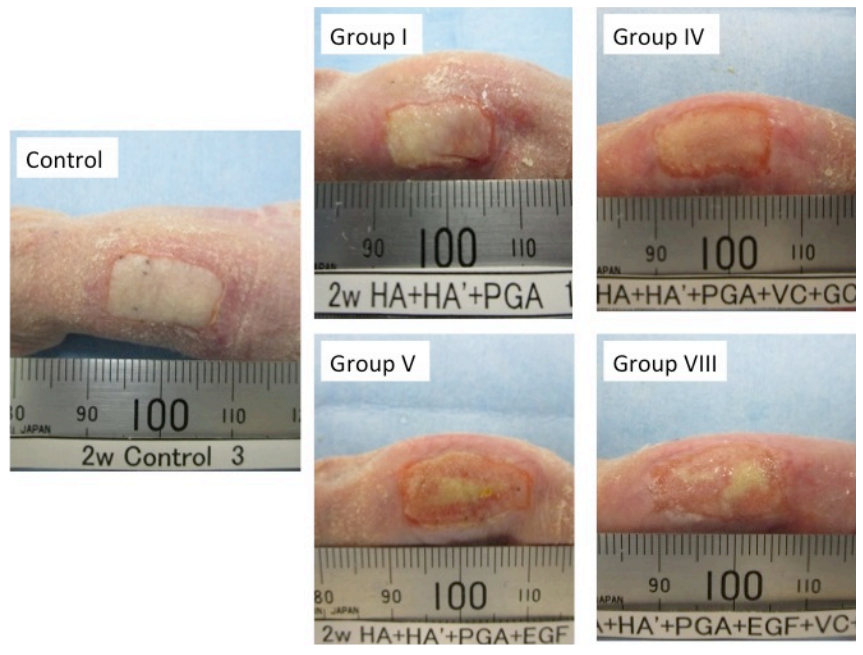

Figure 8. Macroscopic appearances of the wound surface after 2 weeks in the control group (no spongy sheet), Group I, Group IV, Group V, and Group VIII. 


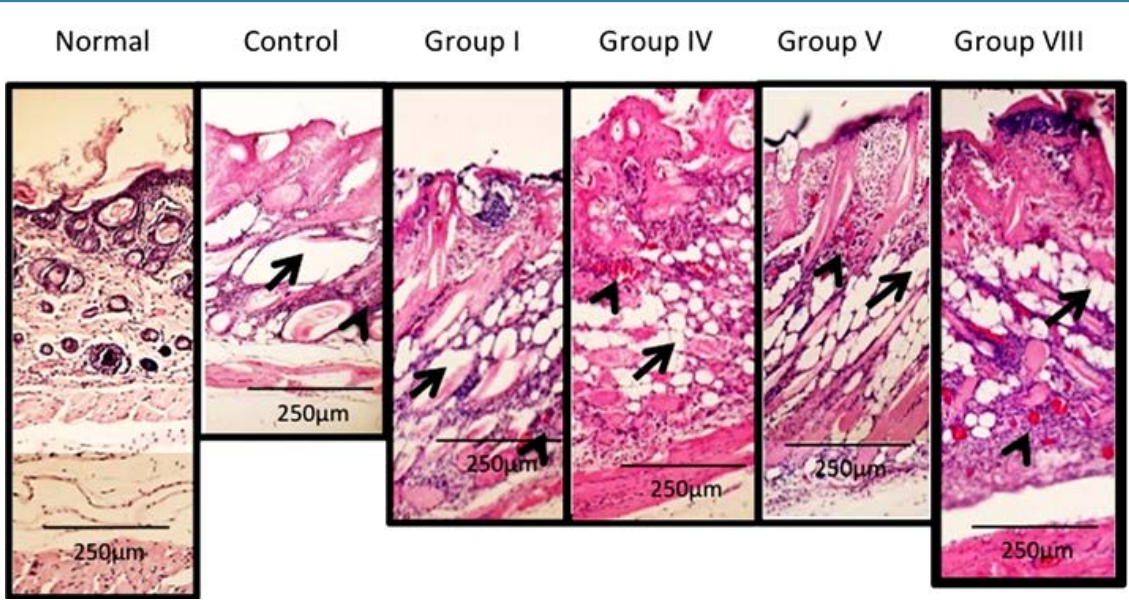

Figure 9. Histological appearances (HE staining) of the dermis after 2 weeks in the control group (no spongy sheet), Group I, Group IV, Group V, and Group VIII. Arrows indicate edema. Arrowheads indicate vascularization. Scale bar $=250 \mu \mathrm{m}$. Magnification $\times 100$.

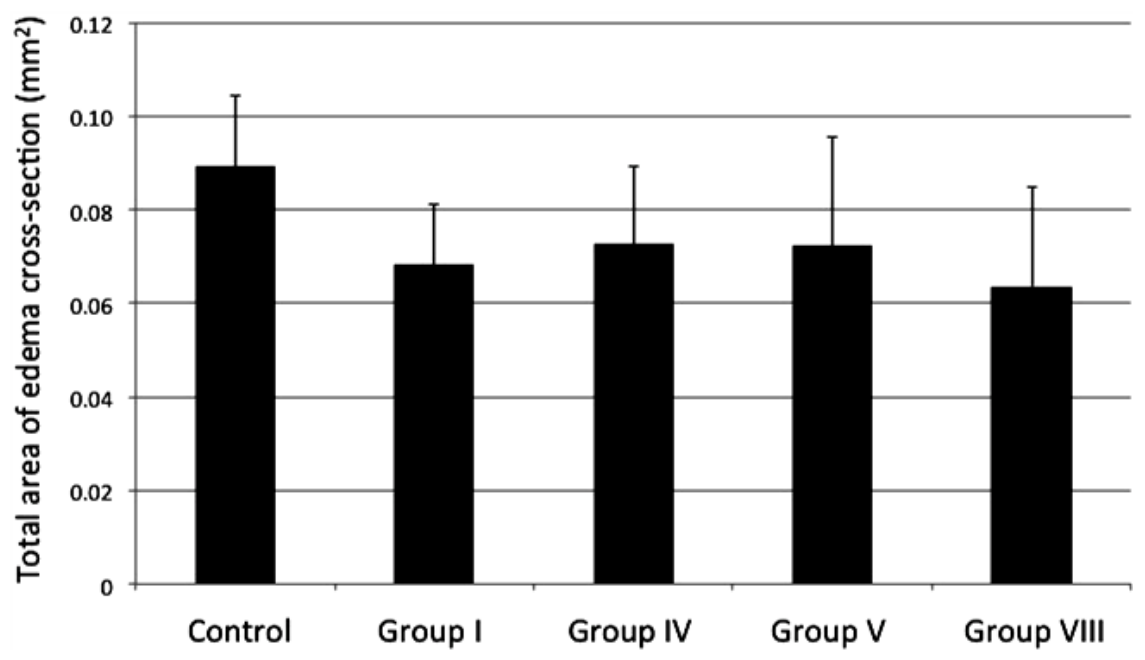

Figure 10. Extent of edema evaluated by measuring the total area of edema in a section of granulation tissue after 2 weeks in the control group, Group I, Group IV, Group V, and Group VIII. $\mathrm{n}=12$.

is due to the destruction of proteins, they resemble thermal burns. This type of injury is referred to as a chemical burn. In general, acid should be washed from the skin surface with water and neutralized with sodium bicarbonate if necessary. On the other hand, the wound surface after chemical peel is washed with water and not neutralized with sodium bicarbonate, because the aim of chemical peel is to cause tissue damage that can be healed. In practice, there is a risk of tissue damage that fails to heal. Therefore, an appropriate skin care product should be used after chemical peels.

In the first experiment, the effects of EGF-free spongy sheets (Groups I-IV) and EGF-incorporating spongy sheets (Groups V-VIII) on fibroblast proliferation were assessed. Based on the results of this preliminary experiment, four types of spongy sheet (Groups I, IV, V, VIII) were assessed in the main experiments using a wound surface model and an animal model. The results of the wound surface model experiment demonstrated that VEGF and HGF production was significantly higher in Group V (HA/PGA + EGF) compared with Group I (HA/PGA alone). This suggests that EGF released from the spongy sheet can more effectively enhance VEGF and HGF production by fibroblasts. In particular, HGF production was higher in Group VIII (HA/PGA + VC, GC, EGF) compared with Group IV (HA/PGA + VC, GC) and Group V. This increase in HGF production appeared to be due to the combined effects of VC, GC and EGF. HGF production was significantly higher than 


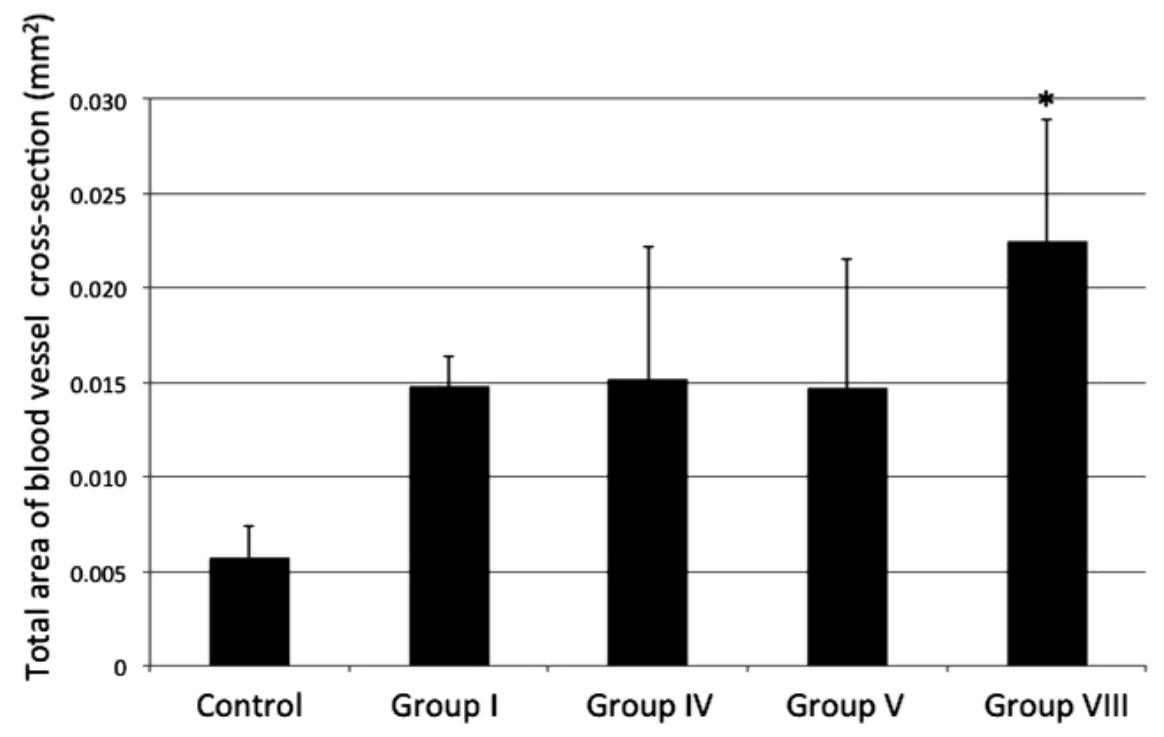

Figure 11. Extent of angiogenesis evaluated by measuring the total number of blood vessels in a section of granulation tissue after 2 weeks in the control group, Group I, Group IV, Group V, and Group VIII. $\mathrm{n}=12$. $^{*} \mathrm{P}<0.01$ vs. Control.

VEGF production in Group VIII. These findings suggest that Group VIII is the most promising aid to facilitate wound healing.

The results in the animal experiment indicate that Group I has the potential to enhance wound healing, and the incorporated bioactive components including VC, GC and EGF have the potential to enhance the wound-healing effect of the spongy sheet. These findings suggest that Group VIII is the most promising aid to facilitate wound healing after chemical peels. In general, deeper peels require longer healing times and are more likely to result in a larger number of complications. The present animal experiment using 50\% TCA is categorized into deeper peels. Therefore, re-epithelialization was not completed within a two-week period. The next study needs an observation during a long period at least more than 1 month.

\section{References}

[1] Wolfort, F.G., Dalton, W.E. and Hoopes, J.E. (1972) Chemical Peel with Trichloroacetic Acid. British Journal of Plastic Surgery, 25, 333-334. http://dx.doi.org/10.1016/S0007-1226(72)80071-7

[2] Baker, T.J., Gordon, H.L., Mosienko, P. and Seckinger, D.L. (1974) Longterm Histological Study of Skin after Chemical Peeling. Plastic and Reconstructive Surgery, 53, 522-525. http://dx.doi.org/10.1097/00006534-197405000-00003

[3] Stough III, D.B. and Irwin, W.G. (1974) Chemical Peel for Facial Wrinkles. American Family Physician, 10, $106-108$.

[4] Coleman III, W.P. and Brody, H.J. (1997) Advances in Chemical Peeling. Dermatologic Clinics, 15, 19-26. http://dx.doi.org/10.1016/S0733-8635(05)70411-3

[5] Dinner, M.I. and Artz, J.S. (1998) The Art of the Trichloroacetic Acid Chemical Peel. Clinics in Plastic Surgery, 25, 53-62.

[6] Brody, H.J., Monheit, G.D., Resnik, S.S. and Alt, T.H. (2000) A History of Chemical Peeling. Dermatologic Surgery, 26, 405-409. http://dx.doi.org/10.1046/j.1524-4725.2000.00505.x

[7] Anitha, B. (2010) Prevention of Complications in Chemical Peeling. Journal of Cutaneous and Aesthetic Surgery, 3, 186-188. http://dx.doi.org/10.4103/0974-2077.74500

[8] Han, S.-H., Kim, H.-J., Kim, S.-Y., Kim, Y.-C., Choi, G.-S. and Shin, J.-H. (2011) Skin Rejuvenating Effects of Chemical Peeling: A Study in Photoaged Hairless Mice. International Journal of Dermatology, 50, 1075-1082. http://dx.doi.org/10.1111/j.1365-4632.2010.04712.x

[9] Kondo, S. and Kuroyanagi, Y. (2012) Development of Wound Dressing Composed of Hyaluronic Acid and Collagen Sponge with Epidermal Growth Factor. Journal of Biomaterials Science, 23, 629-643. http://dx.doi.org/10.1163/092050611X555687

[10] Kondo, S., Niiyama, H., Yu, A. and Kuroyanagi, Y. (2012) Evaluation of a Wound Dressing Composed of Hyaluronic 
Acid and Collagen Sponge Containing Epidermal Growth Factor in Diabetic Mice. Journal of Biomaterials Science, 23, 1729-1740.

[11] Chen, W.Y.J. and Abatangelo, G. (1999) Functions of Hyaluronan in Wound Repair. Wound Repair and Regeneration, 7, 79-89. http://dx.doi.org/10.1046/j.1524-475X.1999.00079.x

[12] Pardue, E.L., Ibrahim, S. and Ramamurthi, A. (2008) Role of Hyaluronan in Angiogenesis and Its Utility to Angiogenic Tissue Engineering. Organogenesis, 4, 203-214. http://dx.doi.org/10.4161/org.4.4.6926

[13] West, D.C., Hampson, I.N., Arnold, F. and Kumar, S. (1985) Angiogenesis Induced by Degradation Products of Hyaluronic Acid. Science, 228, 1324-1326. http://dx.doi.org/10.1126/science.2408340

[14] Lees, V.C., Fan, T.P. and West, D.C. (1995) Angiogenesis in a Delayed Revascularization Model Is Accelerated by Angiogenic Oligosaccharides of Hyaluronan. Laboratory Investigation, 73, 259-266.

[15] Carpenter, G. and Cohen, S. (1976) Human Epidermal Growth Factor and the Proliferation of Human Fibroblasts. Journal of Cellular Physiology, 88, 227-237. http://dx.doi.org/10.1002/jcp.1040880212

[16] Carpenter, G. and Cohen, S. (1979) Epidermal Growth Factor. Annual Review of Biochemistry, 48, $193-216$. http://dx.doi.org/10.1146/annurev.bi.48.070179.001205

[17] Yu, A., Matsuda, Y., Takeda, A., Uchinuma, E. and Kuroyaangi, Y. (2012) Effect of EGF and bFGF on Fibroblast Proliferation and Angiogenic Cytokine Production from Cultured Dermal Substitutes. Journal of Biomaterials Science, Polymer Edition, 23, 1315-1324.

[18] Xin, X., Yang, S., Ingle, G., Zlot, C., Rangell, L., Kowalski, J., Schwall, R., Ferrara, N. and Gerritsen, M.E. (2001) Hepatocyte Growth Factor Enhances Vascular Endothelial Growth Factor-Induce Angiogenesis in Vitro and in Vivo. The American Journal of Pathology, 158, 1111-1120. http://dx.doi.org/10.1016/S0002-9440(10)64058-8

[19] Conway, K., Price, P., Harding, K.G. and Jiang, W.G. (2006) The Molecular and Clinical Impact of Hepatocyte Growth Factor, Its Receptor, Activators, and Inhibitors in Wound Healing. Wound Repair and Regeneration, 14, 2-10. http://dx.doi.org/10.1111/j.1524-475X.2005.00081.X

[20] Niiyama, H. and Kuroyanagi, Y. (2014) Development of Novel Wound Dressing Composed of Hyaluronic Acid and Collagen Sponge Containing Epidermal Growth Factor and Vitamin C Derivative. Journal of Artificial Organs, 17, 8187. http://dx.doi.org/10.1007/s10047-013-0737-x 
Scientific Research Publishing (SCIRP) is one of the largest Open Access journal publishers. It is currently publishing more than 200 open access, online, peer-reviewed journals covering a wide range of academic disciplines. SCIRP serves the worldwide academic communities and contributes to the progress and application of science with its publication.

Other selected journals from SCIRP are listed as below. Submit your manuscript to us via either submit@scirp.org or Online Submission Portal.
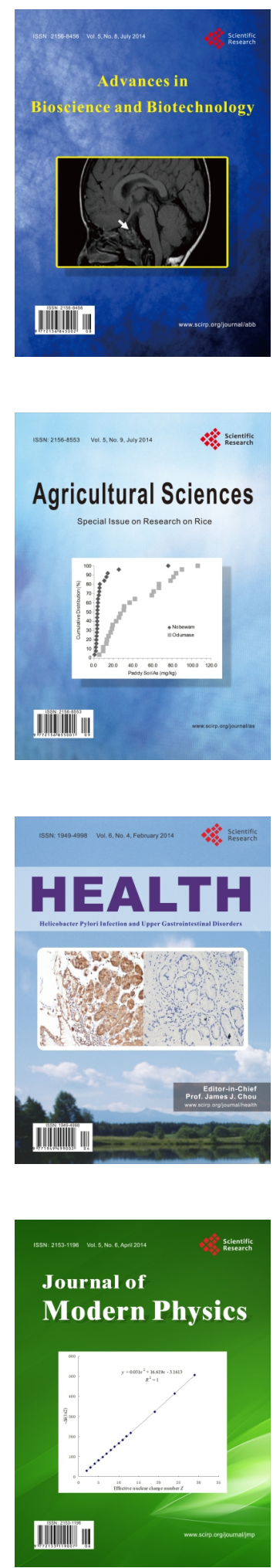
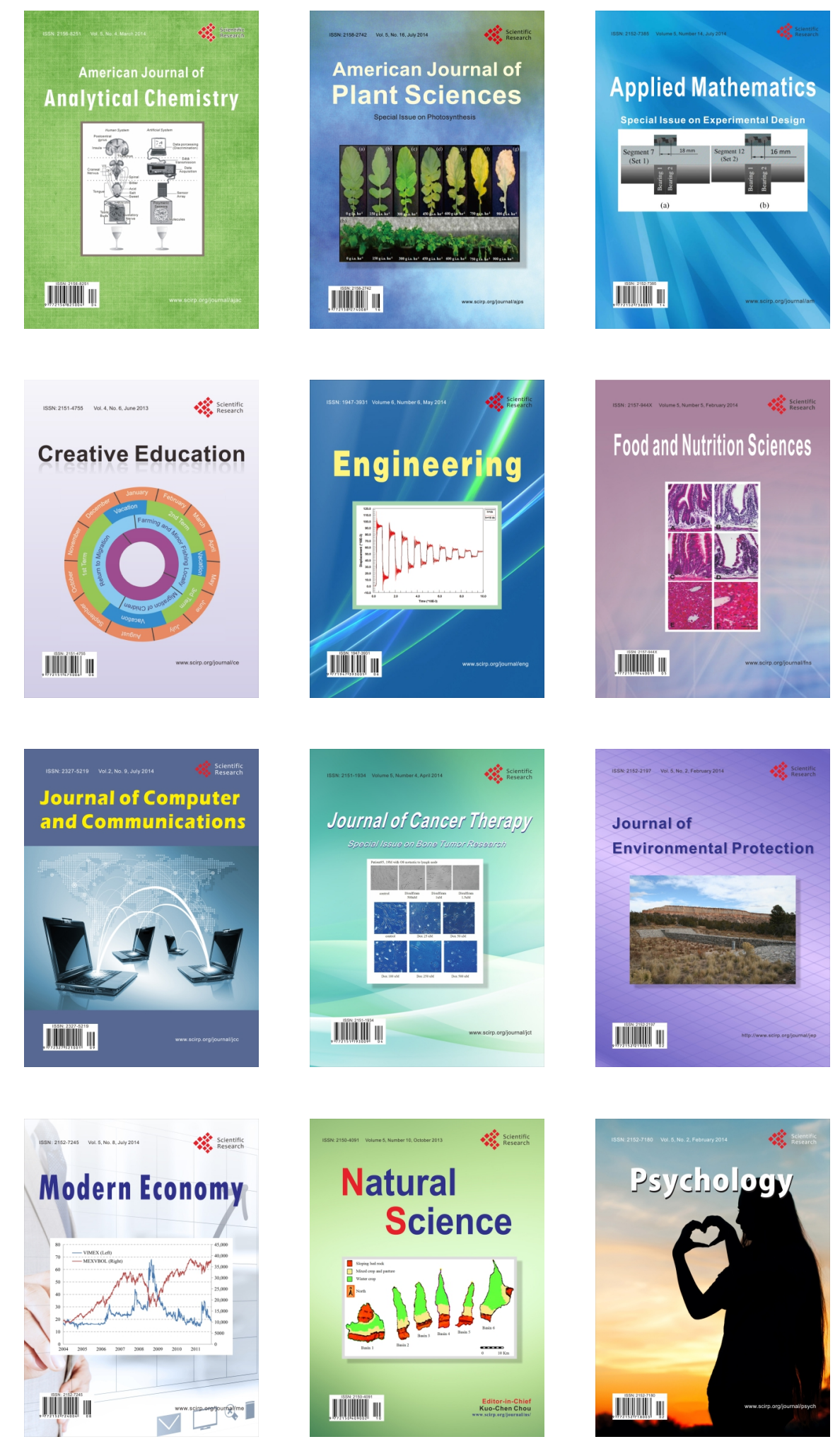